\title{
Path generation modelling: experimentation on Italian road freight transport
}

\author{
A. Quattrone \\ LAST - Laboratory for Transport Systems Analysis, \\ Mediterranea University of Reggio Calabria, Italy
}

\begin{abstract}
In the last decade the considerable growth in European freight transport has almost entirely occurred by road. This increases the importance of understanding and modelling the path choice behaviour of truck-drivers. For explicit path choice modelling two conceptual steps are considered in the literature: 1 . generation of the choice set, that is the possible alternatives, and 2. choice among the alternatives belonging to the given choice set. The first phase is particularly relevant in the path choice process where a large number of alternatives are available between each origin and destination in the network. Starting from the consideration that only a subset of all the possible paths (choice set) are actually perceived by truck-drivers, in this paper a specification, calibration and validation of a path generation model is proposed. The choice set generation is realized with the selective multi-criteria approach, explicitly simulating path perception by truck-drivers in an extra-urban network. The path generation model was calibrated and validated on the Italian road network and also using a truck-driver (road-side and commercial vehicle on-board) survey.
\end{abstract}

Keywords: path choice, path generation and perception, road freight transport.

\section{Introduction}

Freight transport plays a fundamental role in the economy of every country. In the last decade the considerable growth in freight transport in European countries has almost entirely occurred by road. Figure 1 shows the percentage share of road freight in total inland freight transport, expressed in tonne-kilometres (tkm).

Given the high road share of freight transport in Italy and the large number (approximately 4 million) of commercial vehicles in Italy registered in 2004 
(ACI-Italian Automobile Club), the study of truck-drivers' path choice behaviour becomes important to calculate the performance for every element of the supply system.

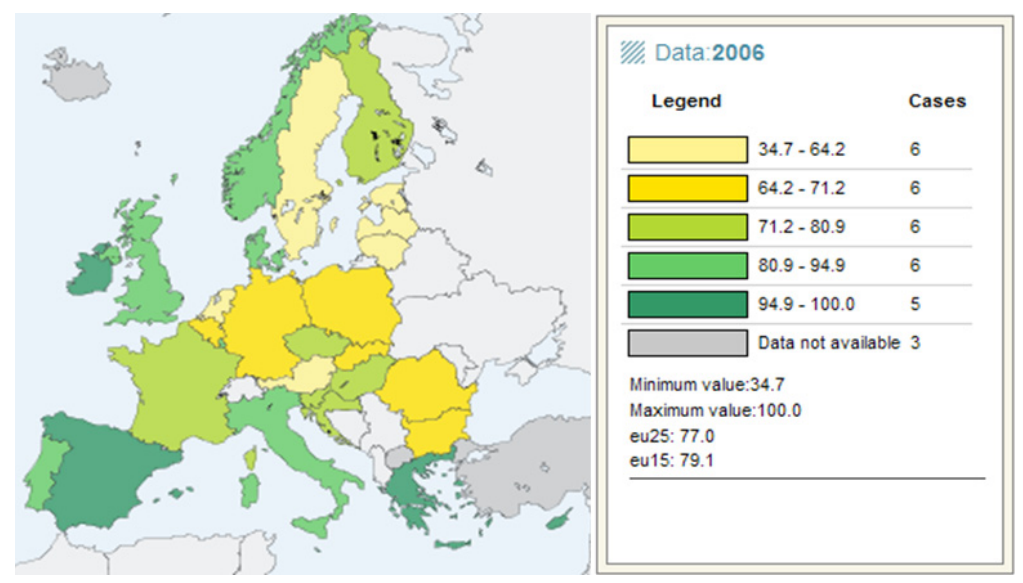

Figure 1: Road share of EU inland freight transport expressed as a percentage of tonne-km (source: Eurostat, 2006).

Starting from the consideration that only a subset of all the possible paths (choice set) is actually perceived by the users, in the literature the problem of path choice has been treated in two phases:

1. generation of the choice set, that explicitly identifies the possible alternatives $[1,2,8,10]$;

2. path choice among the alternatives belonging to the choice set $[1,2,4,5,11]$.

As regards explicit choice set generation an extended literature review is reported in section 2 .

In alternative to explicit approach, the problem of path perception is simulated implicitly in the choice model. This is generally carried out by introducing a perception/availability degree of each alternative through an attribute in the alternatives' utility function [5] or simulated considering the choice set as a fuzzy set in which each alternative has a degree of membership to it $[6,7,9]$.

However, in the literature few behavioural path generation models which also take account of the important process of path perception have been entirely tested for road freight transport.

In this work a path generation model is specified, calibrated and validated. Path generation is simulated with an explicit approach in a separate model: paths are generated with a selective multi-criteria approach, in which for each $(o, d)$ pair the choice set consists of a few paths, each of which is generated by optimizing a covered function associated to a certain criterion (e.g. minimum travel time, maximum motorway use, minimum travel cost etc.). The covered function is calibrated with the maximization of overlapping factors of chosen and generated paths. 
The path generation model was calibrated on a truck-drivers' road-side survey. In all 280 interviews were conducted. The calibration results were validated by applying the model to another database obtained by equipping some truck-drivers' vehicles with on-board GPS (Global Positioning Systems). The utilization of GPS supplies 52 new chosen paths.

Computations of path generation and level-of service-attributes were carried out using the national Italian road network which consists of all the motorways and the main national roads. For the validation formal and informal tests were carried out on parameters and statistics concerning model goodness of fit.

The paper is structured as follows: in section 2 the literature on path choice models is surveyed; section 3 treats the main features of the proposed model; section 4 gives the experimental results, obtained by applying the proposed model to the Italian road transport network; in section 5 some conclusive considerations are reported.

\section{Path choice models}

In the specification of a transport system model, assignment models assume a very important role. They concur to simulate the interaction between supply and demand transport, to calculate user flows and the performance for every element of the supply system. The flows are the result of choice behaviours of those who use the transport system, and in particular they depend on path choice from the zone of origin to destination. The path choice model gives the probability of choosing each path from those perceived as admissible.

Given the trip characteristics of origin and destination, trip purpose, time slice and trip mode, the problem of path choice, for a user travelling between an origin-destination pair $(o, d)$, consists in characterizing "the best" path with various criteria. The best path minimizes trip disutility, which existing assignment models in the literature measure through single attributes, like travel time or distance, or through formulas of generalized travel cost. The problem of path choice is very complex due to the large number of existing alternatives between each $(o, d)$ pair, also on networks of modest dimensions, and their overlapping. These difficulties are treated in various studies $[2,5,10]$ in which complete model specification of path choice is articulated in two phases:

1. generation of the choice set, which explicitly identifies the possible alternatives $[1,2,8,10]$;

2. path choice among the alternatives belonging to the choice set $[1,2,4,5,11]$.

Manski defined in 1977 the probability $p^{n}(k)$ of a generic path $k$ for user $n$ as the sum, on all the choice sets $I_{i}^{n}$ which contain the alternative $k$, of the product between the probability of the choice set $I_{i}^{n}$ and the conditional probability of choosing path $k$ given the choice set $I_{i}^{n}$.

where

$$
p^{n}(k)=\Sigma_{\mathrm{i}} p^{n}\left(I_{i}^{n}, k\right)=\Sigma_{\mathrm{i}} p^{n}\left(I_{i}^{n}\right) \cdot p^{n}\left(k / I_{i}^{n}\right)
$$

- $\quad p^{n}\left(I_{i}^{n}, k\right)$, is the probability of the choice set and the generic path $k$;

- $\quad p^{n}\left(I_{i}^{n}\right)$, is the probability of the choice set $I_{i}^{n}$; 
- $\quad p^{n}\left(k / I_{i}^{n}\right)$, is the conditional choice probability of the generic path $k$ given the choice set $I_{i}^{n}$.

As regards the choice set generation, there are different approaches in the literature (figure 2): in an exhaustive approach, all the loopless analytical paths on the network are available and belong to the single choice set for all users; in a selective approach, only some available paths represent attractive choice alternatives. In the second case, the generation of available paths can be obtained following three different approaches: with the monocriterion approach, deterministic or probabilistic multi-criteria approach.

Regarding the monocriterion approach, the available paths are obtained by the satisfaction of a single criterion. In order to generate the minimum path for the criterion it is necessary to minimize trip disutility measured with a single attribute (time, distance, etc.) or construct a covered function of the criterion. The parameters of the covered function must be calibrated, maximizing, for example, the overlapping factor between the generated and the chosen paths.

Alternatively, it is possible to use the multi-criteria approach according to which the available paths (called "label paths", [2]) are obtained by satisfying some criteria calibrated by maximizing the overlapping factor between the generated and chosen paths, similar to the monocriterion approach.

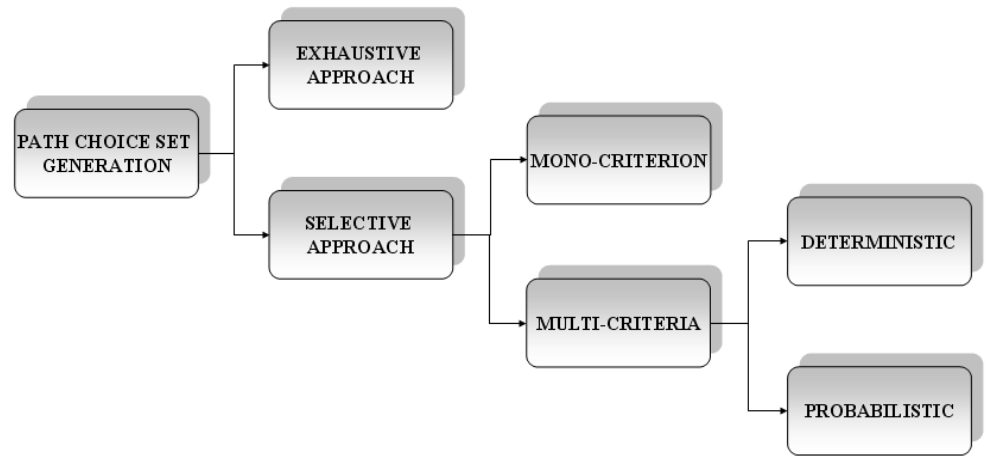

Figure 2: Approaches for choice set generation.

As regards path choice, most of the models proposed in the literature belong to the family of Random Utility Models (RUMs). Based on assumptions on the random residual of the utility perceived by users, the models can have different specifications, of which those most commonly used for path choice are Multinomial Logit, C/DC-Logit, Path Size and Probit [3,5,11,13,14].

\section{Proposed model}

In this paper the first phase of the classical explicit approach for simulating the path choice process is considered: a path generation model is specified, calibrated and validated in order to simulate the perception of a path choice set of a generic user when a large number of alternatives are available. 
Reference was made to previous findings reported in the literature with respect to the behaviour assumed by users in path choice and the factors that determine them. We took into account the fact that travel time and distance are not the only determining factors in the choice process: the user is often induced to choose a path for other reasons, such as trip cost, the level of road safety (i.e. users can choose a certain path as it has low road accident levels), the scenic attraction of the path or the commercial development along it (i.e. users may prefer roads along which there are service areas, bars and restaurants, etc.), and road quality (a path can be attractive due to low winding levels etc.).

Moreover, users on long distances are inclined to prefer the use of motorways, on which they are likely to have more information from personal experience and from any advanced information systems (e.g. VMS - Variable Message Signal), compared with the local road network of which knowledge may be limited.

Finally, meteorological conditions can affect path choice. At certain times of the year, users may prefer coastal and level roads, rather than winding roads that cross mountain zones affected by adverse meteorological conditions.

\subsection{Specification}

The selective approach followed with multicriteria, described in section 2 , is used to define for every $(o, d)$ pair a choice set that consists of several paths $k$, each of which is generated in respect to a certain criterion. Starting from the factors that were assumed to most influence truck-drivers' behaviour in the dimension of path choice, the criteria were defined and optimized to generate suitable paths for the choice set.

These criteria $(c)$ are:

1. minimum path length $(\operatorname{MinL})$;

2. minimum winding $(\operatorname{Min} W)$;

3. minimum path travel time (MinTT);

4. minimum path monetary cost $(\operatorname{MinMC})$;

5. maximum motorway path (MaxM);

6. maximum motorway path with service areas $(\operatorname{Max} S A)$;

7. maximum motorway path with parking areas (MaxPA);

8. maximum motorway path with bars and restaurants $(\operatorname{Max} B \& R)$;

9. minimum motorway path with bridges and viaducts $(\operatorname{Min} B \& V)$;

10. minimum path with high levels of road accidents $(\operatorname{MinR} A)$;

11. minimum path with adverse meteorological conditions (MinAMC).

\subsection{Calibration}

For all the criteria $c$, relative to every link $i$ of the network, we define a covered function equal to the weighted sum of travel time and an attribute that characterizes the same criterion. The function is dependent on unknown parameters that must be calibrated in order to maximize the degree of cover of the paths chosen with those generated (label paths). 
The general structure characterized for the covered functions $c f$ for every criterion $c$ is:

where

$$
\begin{array}{ll}
c f_{i c}=\beta_{m i} A_{i}+\alpha_{m c} B_{i c} & \text { if the link is motorway } \\
c f_{i c}=\beta_{i} A_{i}+\alpha_{c} B_{i c} & \text { otherwise }
\end{array}
$$

- $\quad \beta_{m i}, \beta_{i}, \alpha_{m c}, \alpha_{c}$ are the parameters calibrated for every criterion c;

- $A_{i}, B_{i c}$ are the attributes

$A_{i}, B_{i c}$ attributes are specified in table 1 , which also describes the parameters relative to the covered functions with the above general structure.

Table 1: $\quad$ Parameters of the covered functions for criteria.

\begin{tabular}{lccccccc}
\hline & & Attributes & & \multicolumn{3}{c}{ Parameters } \\
Criterion (c) & $A_{i}$ & $B_{i c}$ & $N_{i c}$ & $\beta_{m i}$ & $\alpha_{m c}$ & $\beta_{i}$ & $\alpha_{c}$ \\
\hline 1. MinL & $\mathrm{L}_{\mathrm{i}}$ & - & - & 1 & 0 & 1 & 0 \\
2. MinW & $\mathrm{t}_{0 \mathrm{i}}$ & $\mathrm{N}_{\mathrm{ic}} \mathrm{L}_{\mathrm{i}} / \mathrm{L}$ & $\mathrm{N}_{\mathrm{Tun}}$ & 1 & to calibrate & 10 & to calibrate \\
3. MinTT & $\mathrm{t}_{0 \mathrm{i}}$ & 0 & - & 1 & 0 & 1 & 0 \\
4. MinMC & $\mathrm{t}_{0 \mathrm{i}}$ & $\mathrm{FC}_{\mathrm{i}}+\mathrm{Toll}_{\mathrm{i}}$ & - & 1 & to calibrate & 10 & to calibrate \\
5. MaxM & 0 & $\mathrm{t}_{0 \mathrm{i}}$ & - & 1 & 1 & 1 & to calibrate \\
6. MaxSA & $\mathrm{t}_{0 \mathrm{i}}$ & $\mathrm{N}_{\mathrm{ic}} \mathrm{L}_{\mathrm{i}} / \mathrm{L}$ & $\mathrm{N}_{\mathrm{SA}}$ & 1 & to calibrate & 10 & to calibrate \\
7. MaxPA & $\mathrm{t}_{0 \mathrm{i}}$ & $\mathrm{N}_{\mathrm{ic}} \mathrm{L}_{\mathrm{i}} / \mathrm{L}$ & $\mathrm{N}_{\text {Parch }}$ & 1 & to calibrate & 10 & to calibrate \\
8. MaxB\&R & $\mathrm{t}_{0 \mathrm{i}}$ & $\mathrm{N}_{\mathrm{ic}} \mathrm{L}_{\mathrm{i}} / \mathrm{L}$ & $\mathrm{N}_{\mathrm{B} \& \mathrm{R}}$ & 1 & to calibrate & 10 & to calibrate \\
9.MinB\&V & $\mathrm{t}_{0 \mathrm{i}}$ & $\mathrm{N}_{\mathrm{ic}} \mathrm{L}_{\mathrm{i}} / \mathrm{L}$ & $\mathrm{N}_{\mathrm{P} \& \mathrm{~V}}$ & 1 & to calibrate & 10 & to calibrate \\
10.MinRA & $\mathrm{t}_{0 \mathrm{i}}$ & $\mathrm{t}_{0 \mathrm{i}} \mathrm{I}_{\mathrm{i}}$ & - & 1 & to calibrate & 1 & to calibrate \\
11.MinAMC & 0 & $\mathrm{t}_{0 \mathrm{i}}$ & - & 1 & to calibrate & 1 & to calibrate \\
\hline
\end{tabular}

$A_{i}, B_{i c}$ attributes in particular depend on:

- $\quad N_{i c}$ that is, relative to each criterion, number of tunnels $\left(N_{T u n}\right)$, bridges and viaducts $\left(N_{B \& V}\right)$, service areas $\left(N_{S A}\right)$, parking areas $\left(N_{\text {Parch }}\right)$ or bars and restaurants $\left(N_{B \& R}\right)$, existing on the motorway to which link $i$ belongs, with respect to the type of specific criterion $c$;

- $\quad L$, that is the total length of motorway to which link $i$ belongs;

- $\quad L_{i}$, that is the length of link $i$.

- $t_{0 i}$, that is the travel time calculated by using functions reported in the literature: functions proposed by the Italian National Research Council (1983) were used for motorways, while TRRL functions (1980) were used for extra-urban roads;

- Toll $_{i}$, that is the toll monetary cost $[€]$ estimated as the product of the link's length $[\mathrm{km}]$ and unit cost per kilometre assumed equal to 0.05 $[€ / \mathrm{km}]$;

- $\quad F C i$, that is the cost of fuel consumption estimated by Russo (2005) as the product of the link's length, unit fuel consumption in litres per kilometre $[1 / \mathrm{km}]$ calculated with the functions proposed by the Italian National Research Council (1983), and the fuel unit cost per litre assumed equal to $0.48 € / 1$; 
- $\quad I i$, that is the number of accidents taking place in 2001 (number of incidents per kilometre divided by the length in $\mathrm{km}$ of the road to which the link belongs).

\section{Experimentation}

The proposed model, described in the previous section, is specified, calibrated and validated for a road truck users' segment, studied with regard to path choice on a road transport network at national scale.

The path generation model was calibrated on a truck-drivers' road-side survey. The chosen path was indicated in the questionnaire through origin, destination and intermediate nodes. In all, 280 interviews with a road-side survey were conducted. In order to get more information about user path choice behaviour and to validate with a different database the parameters calibrated, the vehicles of some truck-drivers were equipped with on-board Intelligent Transportation Systems (ITS) for monitoring freight transport (GPS-Global Positioning Systems). The utilization of GPS supplies new real time data about path choice. In all 52 chosen paths were monitored with on-board vehicle surveys.

Computations of path generation and level-of service-attributes were carried out using the Italian national road network which consists of all the motorways and the main national roads (the network consists of 4480 nodes and 16029 links).

The data for each network link concern the physical and functional characteristics (i.e. length, width and number of lanes, capacity, type of link, winding etc.). Once the number of service areas, tunnels, bridges and viaducts, parking areas, bars and restaurants on the motorway network were known, the attributes $N_{i c}$, required to specify the covered functions, were calculated as described in section 3 .

The set of possible alternatives was defined on the basis of the satisfaction of some criteria, among these defined in the previous section, calibrated by maximizing the overlapping factor between the chosen and the generated paths.

For each considered criterion $c$, the first 30 paths between each $(o, d)$ pair were generated through a $k$ shortest paths algorithm with heap management of the nodes waiting list [13].

The percentage $\lambda_{c g}$ of similarity among the paths chosen by the users in the survey and those generated was calculated with the relation:

where

$$
\lambda_{c g}=2 L_{c g} /\left(L_{c}+L_{g}\right)
$$

- $\quad L_{c g}$, is the length of link shared by both paths (chosen and generated) relative to the same $(o, d)$ pair;

- $\quad L_{c}$, is the total length of the chosen path;

- $L_{g}$, is the total length of the generated path.

Overlapping of a generated path was assumed when its similarity reached $\lambda=$ $90 \%$ with the chosen path. Different combinations of criteria were considered in order to obtain the maximum overlapping between generated and chosen paths 
and to identify the factors that most influence truck-driver behaviour. The same paths generated by different criteria were eliminated (two paths were considered coincident if they differed by less than $5 \%$ of their length).

Considering five of the eleven criteria specified in section 3.1 (table 2) we obtained, through the first (I) and second (II) path generated, overlapping for 216 of the 280 chosen paths within the road-side survey. Considering also the paths generated with all criteria together, only these five criteria give a significant increase in the value of the overlapping factor.

Table 2: $\quad$ Total covered paths (through paths I and II generated) with criteria $3,4,5,9$, and 10 .

\begin{tabular}{|c|c|c|c|c|c|}
\hline \multirow{2}{*}{ Criterion (c) } & \multirow[b]{2}{*}{$\alpha_{c}$} & \multicolumn{2}{|c|}{ Covered Paths } & \multicolumn{2}{|c|}{ Percentage of overlapping } \\
\hline & & I & I and II & I & I and II \\
\hline \multicolumn{6}{|c|}{ ROAD-SIDE SURVEY } \\
\hline 3. $\operatorname{Min} T T$ & - & 136 & 141 & 48.57 & 50.36 \\
\hline 4. $\operatorname{MinMC}$ & $10 \mathrm{~min} / €$ & 137 & 143 & 48.93 & 51.07 \\
\hline 5. $\operatorname{Max} M$ & 1.5 & 144 & 149 & 51.43 & 53.21 \\
\hline 9. $M i n B \& V$ & $12 \mathrm{~min}$ & 197 & 199 & 70.36 & 71.07 \\
\hline 10.MinRA & $\alpha=1^{*}, \alpha=2^{* *}$ & 208 & 216 & 74.29 & 77.14 \\
\hline Total covered $p$ & & 208 & 216 & 74.29 & 77.14 \\
\hline Total non-cove & dpath & 72 & 64 & & \\
\hline Total chosen pc & & & & & \\
\hline \multicolumn{6}{|c|}{$\frac{280}{O N-B O A R D \text { VEHICLE SURVEY }}$} \\
\hline 3. MinTT & - & 28 & 33 & 53.85 & 63.46 \\
\hline 4. $\operatorname{MinMC}$ & $10 \mathrm{~min} / €$ & 29 & 35 & 55.77 & 67.31 \\
\hline 5. MaxM & 1.5 & 29 & 35 & 55.77 & 67.31 \\
\hline 9. $M i n B \& V$ & $12 \mathrm{~min}$ & 32 & 37 & 61.54 & 71.15 \\
\hline 10. MinRA & $\alpha=1^{*}, \alpha=2^{* *}$ & 33 & 38 & 63.46 & 73.08 \\
\hline \multirow{3}{*}{\multicolumn{2}{|c|}{$\begin{array}{l}\text { Total covered paths } \\
\text { Total non-covered path } \\
\text { Total chosen paths }\end{array}$}} & 33 & 38 & 63.46 & 73.08 \\
\hline & & \multirow{2}{*}{\multicolumn{2}{|c|}{52}} & & \\
\hline & & & & & \\
\hline
\end{tabular}

Note: * on motorway link; ** otherwise

Moreover, in order to verify the goodness of calibrated parameters, considering the same five criteria, overlapping between generated and chosen paths was also calculated for the database obtained with the on-board vehicle survey. In table 2 a comparison between the results obtained for both surveys (road-side and on-board vehicle) is reported. The columns relative to the covered paths show cumulated paths obtained with the five ordered criteria: for example, in the case of the road-side survey considering the criterion 5.MaxM (with path I generated), there are seven paths covered in addition to those obtained with the two previous criteria (MinTT and MinMC) $(144-137)$. The same reasoning was made for the percentage of overlapping which is a cumulated value for each criterion.

Table 2 shows that the good percentage of overlapping obtained for the first database of the road-side survey (74.29-77.14\%) is drawn again for the second database of the on-board vehicle survey $(63.46-73.08 \%)$, taking into account that the two databases have different sizes.

Finally, in table 3 a matrix is reported in which the generic element $a_{i j}$ supplies, if $i=j$, the number of chosen paths covered (with the first generated) 
with the criterion $i$ (or $j$ ), while if $i \neq j$, the number of chosen paths covered with the criterion $i$ in addition to those generated wit the criterion $j$. The matrix is relative to both databases (road-side and on-board vehicle survey).

Table 3: $\quad$ Total covered paths through the first generated paths.

\begin{tabular}{cccccc}
\hline \multicolumn{7}{c}{ Criterion (c) } \\
\hline Criterion (c) & MinTT & MinB\&V & MinMC & MaxM & MInRA \\
\hline MinTT & $136(28)$ & $189(31)$ & $168(32)$ & $162(30)$ & $152(29)$ \\
MinB\&V & $189(31)$ & $131(29)$ & $134(31)$ & $164(30)$ & $148(32)$ \\
MinMC & $168(32)$ & $134(31)$ & $97(31)$ & $145(31)$ & $114(33)$ \\
MaxM & $162(30)$ & $164(30)$ & $145(31)$ & $130(9)$ & $142(30)$ \\
MInRA & $152(29)$ & $148(32)$ & $114(33)$ & $142(30)$ & $33(27)$ \\
\hline
\end{tabular}

Note: $(.)=$. relative to the on-board vehicle survey database

\section{Conclusions}

In this paper, a model explicitly simulating path perception by truck-drivers is proposed. Path choice set generation was carried out with a selective multicriteria approach. This approach is able to identify a subset (choice set) of a few paths among all the available alternatives between each $(o, d)$ pair, in agreement with the real behaviour of a generic user who does not perceive all the paths but just the paths that satisfy some criteria. The choice set was defined on the basis of the optimization of a covered function associated to a specific criterion by maximizing the overlapping factor between the chosen and generated paths. Some calibration results, based on the observation of the behaviour of a sample of truck-drivers that use the Italian road network, are also presented.

The experiment carried out on the Italian network confirms that: few routes are actually perceived as feasible alternatives; a significant degree of coverage ( $80 \%$ about) of chosen paths could be obtained by generating paths with respect for the criteria identified; topological, level of service and socio-economic attributes influence users' perception. Although the results presented are to be considered preliminary, they demonstrate the feasibility of the proposed model as a tool for quantitative evaluation of factors influencing truck-driver path perception. In the future, similar models should be calibrated on larger samples from several market segments and the generation choice set phase should be integrated with the consequent phase of path choice simulation in network assignment models.

The properties of the model should also be further analyzed in order to enable a comparison between implicit and explicit generation. The model could be completed with the simulation of the path choice among the alternatives belonging to the choice set and integrated within a path choice simulation procedure in network assignment models (further development). 


\section{References}

[1] Antonisse, R. W., Daly, A. J., and Ben Akiva, M., Highway assignment method based on behavioural models of car driver's route choice, Transportation Research Record (1220), pp. 1-11, 1985.

[2] Ben Akiva, M., Bergman M. J., Daly A. J., Ramaswamy, R., Modelling interurban route choice behaviour, Proceedings of the $9^{\text {th }}$ International Symposium on Transportation and Traffic Theory, VNU Science Press, pp. 299-330, 1984.

[3] Cantarella, G. E., Vitetta, A., The multi-criteria road network design problem in an urban area. Transportation, vol. 33, pp. 357-588, 2006.

[4] Cascetta, E., Nuzzolo, A., Biggiero, L., Analysis and modelling of commuters departure time and route choices in urban networks, Proceedings of the $2^{\text {nd }}$ International Seminar on Urban Traffic Network, Capri, Italy, 1992.

[5] Cascetta, E., Nuzzolo, A., Russo, F., Vitetta, A., A new route choice logit model overcoming IIA problems: specification and some calibration results for interurban networks, Proceedings of the $13^{\text {th }}$ International Symposium on Transportation Traffic Theory, Jean-Baptiste Lesort ed., Pergamon Press, 1996.

[6] Cascetta, E. and Papola, A., Random utility models with implicit availability/perception of choice alternatives for the simulation of travel demand, Transportation Research Part C 9, pp. 249-263, 2001.

[7] Cascetta, E., Russo, F., Viola, F.A., Vitetta, A., A model of route perception in urban road networks, Transportation Research Part B 36, pp. 577-592, 2002.

[8] Morikawa, T., A hybrid probabilistic choice set model with compensatory and noncompensatory ruler, Proceedings of the $7^{\text {th }}$ WCTR, Sydney, Australia, 1996.

[9] Russo, F., Fuzzy Theory in Transportation Field: Fuzzy Sets for Simulating Path Choice Behaviour, in Advanced in Intelligent Systems, Morabito F.C. (ed.), IOS Press, 1997.

[10] Russo, F. and Vitetta, A., Networks and assignment models for the Italian national transportation system, Proceedings of the $7^{\text {th }}$ WCTR, Sydney, Australia, 1995.

[11] Russo, F. and Vitetta, A., An assignment model with modified Logit, which obviates enumeration and overlapping problems, Transportation 30, pp. 117-201, 2003.

[12] Russo, F., Vitetta, A., La ricerca dei percorsi in una rete: algoritmi di minimo costo ed estensioni. FrancoAngeli, Milan, Italy, 2006.

[13] Russo, F., Vitetta, A., Quattrone, A., Route choice modelling for freight transport at national level. Proceedings of the European Transport Conference, Strasbourg, 2007.

[14] Sheffy, Y., Urban transportation networks, Prentice Hall, Englewood Cliff, NJ, 1985. 